\title{
Acuerdos de Paz de Esquipulas: La construcción de la paz en centroamérical
}

Por Soren Chamorro ${ }^{2}$

Recibido: 20.10.2015 / Aprobado: 3.11.2015

\section{RESUMEN}

El presente artículo tiene como finalidad realizar una mirada general al proceso de Paz de Centroamérica, después de una de las etapas más conflictivas de su historia. Se repasan algunas de las características particulares que llevaron a cada uno de estos países a tomar las armas, pero así también, aquellas medidas de solidaridad internacionales y nacionales que contribuyeron con la firma de los Acuerdos de Esquipulas y los posteriores acuerdos para recuperar y consolidar la paz a lo interno de cada uno de los países centroamericanos.

Palabras clave: Proceso de Paz, Acuerdos de Esquipulas, Centroamérica.

\begin{abstract}
This article is intended to perform a general look at the Peace Process in Central America, after one of the most conflictive stages of its history. It reviews some of the particular characteristics that led each of these countries to take up arms but also those measures of national and international solidarity that contributed to the signing of the Esquipulas Accords and the subsequent agreements to recover and consolidate peace internally in each of the Central American countries.
\end{abstract}

Key words: Peace process, Esquipulas Accords, Central America.

\section{Antecedentes de la Conflictividad en Centroamérica}

Si bien cada uno de los países centroamericanos que experimentaron una guerra civil agravada en los años 80, presentan características particulares que obedecen a sus propios contextos, también podemos encontrar características comunes tales como la prolongada exclusión de grandes sectores de la población, estados militarizados, dictaduras, concentración de riqueza en manos de la oligarquía y de grupos burgueses emergentes, entre otros. Estas condiciones de violencia estructural

1. Contribución del Instituto "Martin Luther King" de la UPOLI, en el evento internacional de investigación sobre la paz, organizado por el Consorcio Internacional de Investigación sobre la Violencia (ICRoV) y realizado en Etiopía en octubre 2015.

2. Máster en estudios Internacionales de Paz, Conflictos y Desarrollo. Responsable de Proyectos y Relaciones Internacionales del Instituto "Martin Luther King" de la Universidad Politécnica de Nicaragua (UPOLI). 
continua, fueron agravadas en el marco del contexto de la guerra fría, donde países como Estados Unidos, Cuba y Rusia intervinieron prestando apoyo logístico y financiero a los gobiernos y/o guerrillas aliados en busca de la desestabilización de sus contrincantes.

Entre los elementos que han sido identificados como las raíces profundas de los conflictos en El Salvador, Guatemala y Nicaragua podemos mencionar los siguientes:

En el Salvador: se puede identificar como las raíces profundas del conflicto las reformas de 1882 realizadas por el gobierno liberal, donde se abolieron las propiedades comunales, a fin de favorecer a un grupo reducido de familias para la producción de café. En los subsiguientes años esta concentración de la tierra se fue agravando y favoreciendo una fuerte influencia de este sector en la conformación del Estado Salvadoreño, quien se veía favorecidos por los altos impuestos que este sector les aportaba. Esta situación fue generando inconformidades en el resto de la población que unida con la crisis económica de 1929 llevó a los primeros estallidos de violencia, destacándose la matanza de 30,000 campesinos en 1931 por el dictador Maximiliano Martínez. A fin de que el gobierno Salvadoreño y las elites económicas pudiesen mantener su status quo, se empezó el proceso de militarización del estado, limitando los pocos espacios democráticos. A finales de los años 70 el Estado Salvadoreño con el apoyo de los Estados Unidos trató de frenar el avance de la lucha popular promoviendo una especie de apertura democrática por medio del desarrollo económico del país y la puesta en marcha de algunas políticas sociales. Sin embargo estas no fueron suficientes para solventar las necesidades de la población que se movilizaba y más bien recibía mayores controles y represión por parte del gobierno, llevando al país a un virtual desarrollo de la insurgencia (Van der Borgh, s/f).

En el caso de Guatemala resulta importante destacar su característica de estado multiétnico, con un $60 \%$ de la población de origen indígena y un $40 \%$ de origen mestizo (Alvarez y Palencia, 2002). No obstante esta mayoría poblacional no ha sido siempre reconocida como sujeta de derechos y por muchos años vivieron bajo la opresión de la sociedad mestiza. Al igual que en El Salvador había una alta concentración de las tierras

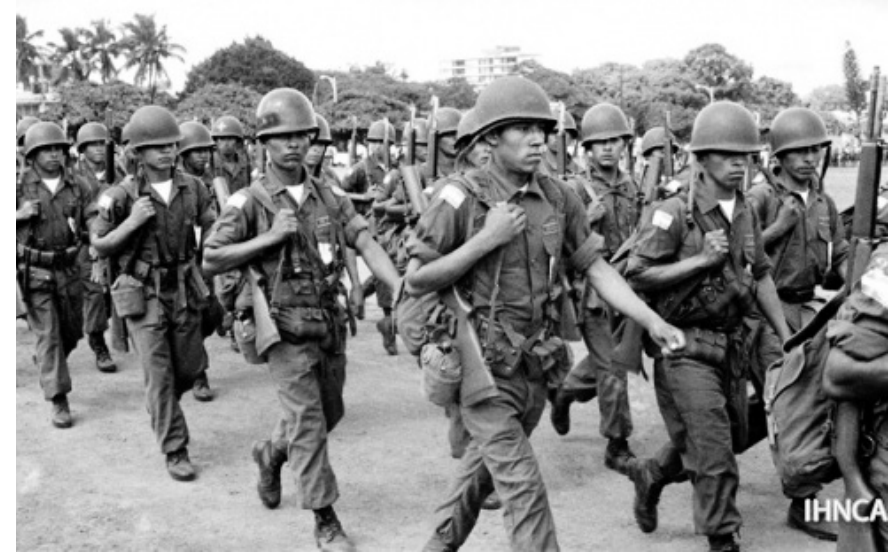

La Guardia Nacional fue el principal instrumento de poder político y control social de la familia Somoza. Diario Novedades, Managua, 1978 Archivo Histórico IHNCA.

fértiles, llegando a ser controladas por el sólo $2.2 \%$ de la población. A esto debemos sumar la coalición entre las élites económicas y los militares, que con sus actuaciones mantenían un orden social inamovible en el que la mayoría de la población no tenía cabida, de tal manera que las elites económicas controlaban las riquezas y los militares el poder. Efectivamente podemos afirmar que en Guatemala hubo un mayor entramado militar, con un pequeño espacio democrático entre 1944 y 1954, bajo el gobierno de Juan Jacobo Árbenz, quien fue derrocado por la intervención de Estados Unidos. Asimismo se destacó por el uso de terrorismo de estado, llegando a cometerse grandes crímenes como el Genocidio Maya suscitado entre 1981 y 1983.

En el caso de Nicaragua, por sus condiciones geoestratégicas, acceso al mar atlántico y pacifico, el país se ha visto marcado por constantes intervenciones, sobre todo de Estados Unidos, apoyando a gobiernos para mantenerse en el poder. Estas relaciones al igual que en El Salvador y Guatemala potenciaron la instauración de entidades militares, tales como la Guardia Nacional en 1927, soporte de la dictadura Somozista por 45 años, la cual se encargó de reprimir a la población, ante sus demandas por la alta concentración de tierras y riquezas en manos de un sector privilegiado y el constante irrespeto hacia los derechos humanos. Ello unido a la crisis económica de 1973, que afectó el desarrollo económico de Nicaragua y la corrupción del régimen en el manejo de los fondos de la cooperación internacional después del terremoto de Managua de 1972, llevo al país a un conflicto armado, resultando en el triunfo de la revolución sandinista en 1979. 
En sus inicios la Revolución Sandinista pensaba poner en práctica un proyecto que se basará en una economía mixta y pluralismo político, no obstante esto nunca pudo lograrse (Matul y Ramírez, S/F). Ya para 1980 el FSLN contaba con una fuerte oposición de grupos que no lograron converger con la política sandinista, quienes con el apoyo de Estados Unidos empezaron una contra revolución.

Todos estos contextos de opresión y acumulación de violencias produjeron una serie de inconformidades en diferentes sectores de las sociedades Centroamericanas, las cuales se fueron reagrupando en torno a diferentes movimientos guerrilleros, que empezaron a hacer frente a los gobiernos que se mostraban como fuentes de esa opresión.

Es importante recalcar que aunque se habla del conflicto Centroamericano no todos los países afrontaban conflictos internos. Sin embargo todos participaban de las dinámicas desarrolladas por los movimientos guerrilleros de los países vecinos. Es así como Costa Rica y Honduras servían de base para los contrarrevolucionarios Nicaragüenses, desde donde hostigaban al gobierno sandinista. Si bien la participación de Honduras fue mayor, tanto Costa Rica como $\mathrm{H}$ ond ura s recibieron cuantiosas ayudas por parte de la Agencia Internacional para el Desarrollo de los Estados Unidos (Hidalgo, 2000).

\section{Proceso de Paz en Centroamérica}

Ya para principios de los años 80 gran parte de los países Centroamericanos estaban inmersos en un proceso de guerra que amenazaba con convertirse en un conflicto regional. Este grado de conflictividad empezó a percibirse como una amenaza para la seguridad, más allá de la región. Esto sumado a un cambio de coyuntura de políticas de países como México, Venezuela, Colombia y Panamá hacia la política de Estados Unidos, dio como resultado el surgimiento de una serie de iniciativas internacionales y regionales de cara a buscar una solución negociada de los conflictos en Centroamérica y por ende la consecución de la paz.

Usualmente se habla del Proceso de Paz en Centroamérica como un conjunto, pero retomando la idea de Alonso Ramírez y Daniel Matul, también sería correcto hablar de varios procesos, todos desarrollados en diversos tiempos y contextos, pero en la línea de conseguir un mismo objetivo, la paz en la región centroamericana.

Tanto Matul como Ramírez insisten en dividir el Proceso de Paz de Centroamérica en tres generaciones de acuerdos de paz.

\section{ACUERDOS DE PRIMERA GENERACIÓN:} Todos los acuerdos desarrollados antes y hasta la Declaración de Esquipulas de 1986. Estos acuerdos estuvieron marcados por iniciativas inter y extra regionales, tal es el caso del grupo de Contadora y el Grupo de Apoyo y donde se encuentran iniciativas tales como, la Resolución de Caraballeda, El Acta de Contadora y la Declaración de Esquipulas I. Los esfuerzos dentro de esta etapa estuvieron enmarcados en la generación de medidas de confianza mutua y en la recolección de las voluntades políticas de los países de Centroamérica de llegar a una solución negociada de los conflictos que llevaran a la región a la consecución de la paz. Si bien durante esta etapa no se obtuvieron resultados tangibles, esta etapa sirvió para enunciar los problemas existentes y para sentar las bases de mecanismos de diálogos tanto regionales como extraregionales.

\section{ACUERDOS DE SEGUNDA}

GENERACIÓN: Esta etapa comprende todos los acuerdos desarrollados dentro de la esfera del Procedimiento para Establecer una Paz Firme y Duradera en Centroamérica, lo cual fue definido en el marco de los Acuerdos de Esquipulas II de 1987. Estos acuerdos establecieron medidas concretas a ser desarrolladas por cada uno de los países centroamericanos y contaban con la posterior verificación de organismos internacionales, tales como la Organización de Naciones Unidas (ONU) y la Organización de Estados Americanos (OEA).

ACUERDOS DE TERCERA GENERACIÓN: Los acuerdos de esta generación se derivan de las dos generaciones anteriores, pero siguen procesos a lo interno de cada país. A diferencia de la segunda generación, las 


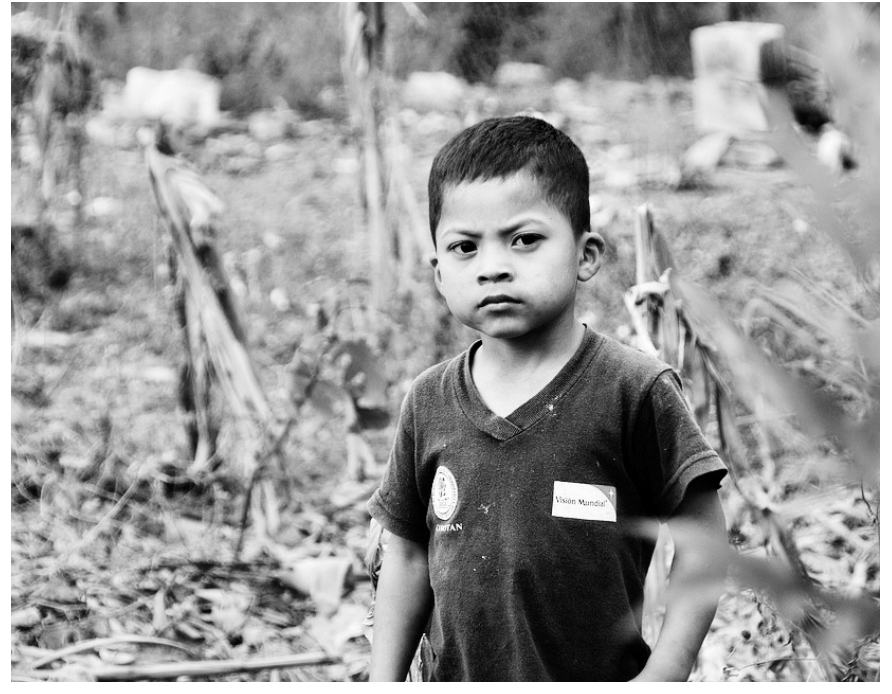

Pixabay.com

negociaciones aquí son desarrolladas entre los grupos enfrentados de forma directa e incluyen procesos más extensos, que revisan las condiciones estructurales que dieron orígenes a los conflictos armados.

Es importante resaltar que cada generación de acuerdos tuvo resultados e impactos diferentes para cada uno de los países centroamericanos involucrados en el conflicto. Si tomamos en cuenta las fechas de la firma de los acuerdos de paz a lo interno de cada país. Nicaragua 1990, El Salvador 1992 y Guatemala 1996, podemos ver que la firma de los Acuerdos de Esquipulas II tuvo un mayor impacto para Nicaragua. No obstante, hubo un mayor desarrollo de los acuerdos de la tercera generación en el caso de El Salvador y Guatemala, quienes requirieron de más tiempo para la firma de los acuerdos de paz.

Cada uno de los acuerdos firmados dentro de este proceso fue un pilar importante para la consecución de la paz. Sin embargo hay algunos que merecen un análisis más detallado y entre ellos resulta indispensable destacar el papel del Grupo de contadora y el Acta que surge al finalizar este proceso; Los Acuerdos de Esquipulas II y los acuerdos claves a lo interno de cada país que llevaron a los países centroamericanos a la firma de la paz.

El Proceso de Contadora fue desarrollado por un periodo de 20 meses, empezando en 1983 y aglomeró a cuatro países latinoamericanos, México, Venezuela, Colombia y Panamá. Fue creado con el objetivo de analizar lo que ocurría en Centroamérica, revisar que propuestas de paz habían sido formuladas hasta entonces y generar propuestas que ayudaran a la consecución de la paz. Una de las grandes sorpresas de este grupo fue la condena hacía los países extra regionales que intervenían en el conflicto Centroamericano (Estados Unidos, Rusia y Cuba), especialmente hacia las políticas de la administración Reagan con respecto a Centroamérica y la convicción manifiesta del grupo de que la conflictividad en Centroamérica no respondía al contexto del conflicto Este - Oeste, sino más bien a las condiciones de desigualdad social y económica en la región (Duran, S/F)

El grupo funcionó como un foro permanente para el dialogo entre los países Centroamericanos y aunque para algunos analistas esta iniciativa fue considerada como un fracaso, en vista de la complejidad de sus propuestas y la oposición manifiesta de Estados Unidos, nada de lo que vino después habría sido posible sin el apoyo de este grupo al proceso de Paz en Centroamérica (Maira, 2014). En este sentido es posible rescatar algunos de los grandes aportes realizados por el grupo de Contadora al proceso de Paz en Centroamérica.

1. Pudo reunir a los 5 países centroamericanos para que ventilaran sus quejas e inquietudes;

2. Se consiguió evitar un conflicto abierto entre Nicaragua y sus países vecinos, Costa Rica y Honduras, logrando de esa manera evitar una escalada bélica en la región;

3. Se definieron los aspectos claves o esenciales para la estabilización de la región;

4. Se establecieron las bases de confianza mutua y mecanismos para posteriores procesos de negociación;

5. Se evitó una intervención militar directa de Estados Unidos en territorio Centroamericano.

El trabajo de este grupo tuvo desde sus inicios respaldo del Consejo de Seguridad, de la Asamblea de Naciones Unidas y de un sinnúmero de organismos regionales e internacionales y para 1985 se incorporó también el Grupos de Apoyo: Perú, Argentina, Brasil, Perú y Uruguay, lo que reafirmaba el compromiso internacional para la búsqueda de una solución al 
conflicto centroamericano. Después de múltiples reuniones y propuestas en 1986 se dio por terminada la tarea del Grupo de Contadora con la entrega del Proyecto de Acta de Contadora para la Paz y la Cooperación en Centroamérica. No obstante, por la complejidad de la misma y el factor gravitante de la política de Estados Unidos en la región, no contó con la aprobación y compromiso de los gobiernos de la región.

\section{Acuerdos de Esquipulas}

Cuando se habla del proceso de Paz en Centroamérica suele darse un salto sustancial entre lo suscitado en el marco del Proceso de Contadora y la firma de los Acuerdos de Esquipulas II. Pero resulta justo retomar lo ocurrido en Esquipulas I, que podría definirse como la plataforma que permitió una transición entre los complejos planteamientos surgidos del Grupo de Contadora y las más pragmáticas medidas planteadas en los Acuerdos de Esquipulas II. Cabe destacar que los acuerdos de Paz de la Segunda generación, plantean una centroamericanización de la paz y de las iniciativas para conseguirla, pues son los mismos presidentes de la región los que facilitan las condiciones para llegar a un fin del conflicto por medio de la negociación.

En el primer semestre de 1986 hay un cambio de gobierno en tres países centroamericanos, en Guatemala asume Vinicio Cerezo, en Honduras José Azcona y Oscar Arias en Costa Rica. Quedando demostrado de esta manera las tendencias de la región a la estabilización y la apertura a gobiernos de tendencia democrática, que en el caso de Costa Rica y Guatemala reafirmaban sus políticas de neutralidad y su deseo de cumplirlas, mejorando notablemente las relaciones con Nicaragua. Estos cambios presidenciales, los fracasos militares de la Contra, el impacto de la mediación ejercida por el Grupo de Contadora y el Grupo de Apoyo, la condena política de Estados Unidos en la Corte Internacional de Justicia, la búsqueda de un acuerdo nuclear entre las súper potencias y el escándalo Irán - Contra, generaron las condiciones ideales para el establecimiento de un nuevo acuerdo, que reavivara las negociaciones entre los países de la región (Aravena, 1989).

Es dentro de este contexto, que los cinco presidentes de los países centroamericanos se reúnen en Esquipulas Guatemala, bajo el auspicio de Vinicio Cerezo
(Presidente de Guatemala en ese entonces) y firman la Declaración de Esquipulas I, en la cual los presidentes de Centroamérica deciden formalizar las reuniones de presidentes como una instancia para analizar las problemáticas de la región en torno a la consecución de la paz y el desarrollo regional. Asimismo se comprometen en generar una cooperación económica y una estructura básica para la resolución pacífica de los conflictos.

Si bien la Declaración de Esquipulas I, pone de manifiesto el interés de los presidentes centroamericanos en llegar a un consenso para la superación de la crisis en la región, todavía no plantea ideas concretas de cómo lograrlo. Es así como después de una serie de reuniones los presidentes de Centroamérica se reúnen nuevamente en Esquipulas y se firman los Acuerdos de Esquipulas II de 1987. El principal contenido de los acuerdos giró en torno al cese de las hostilidades, la democratización, elecciones libres, cese de apoyo para las fuerzas irregulares, control de armas, asistencia a los refugiados y el establecimiento de procedimientos para la verificación internacional sobre el cumplimiento de dichos acuerdos.

Lo realmente novedoso del "Procedimiento para establecer la paz firme y duradera en Centroamérica" o

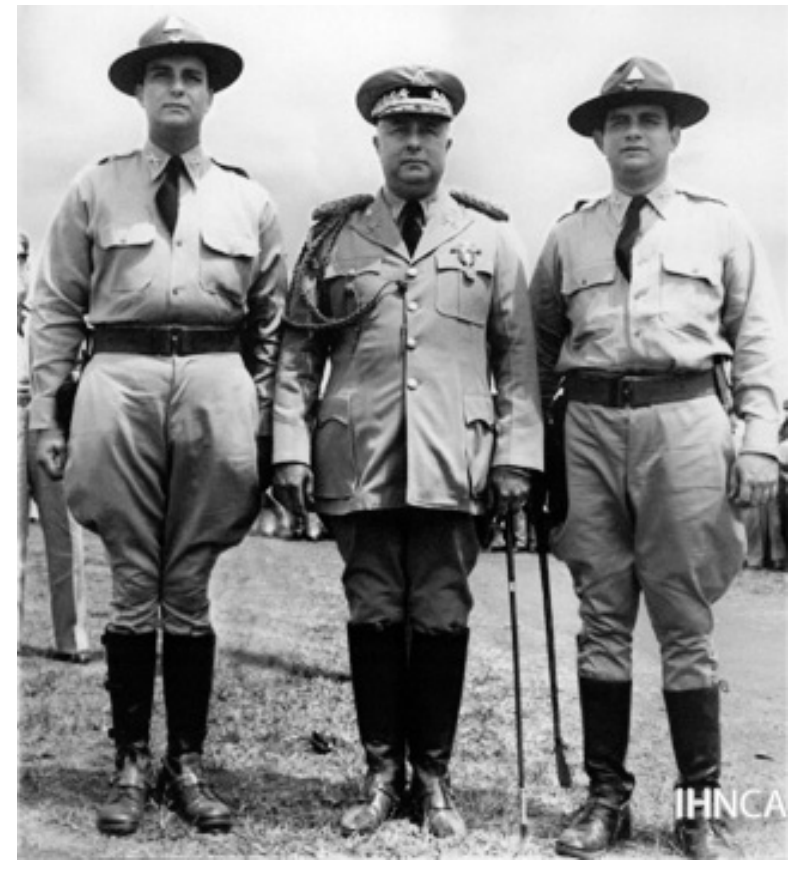

Anastasio Somoza García fundador de la Dinastía Somocista con sus hijos, Luis y Anastasio Somoza Debayle, durante una parada militar en la Explanada de Tiscapa, 1949-50. Talleres Nacionales, Fondo Somoza, Archivo Histórico IHNCA. 
más bien conocido como los Acuerdos de Esquipulas II, con respecto a lo ocurrido con el proceso de Contadora fue:

1. El consenso sobre los conceptos en los cuales estaría basado el proceso. En este caso la paz, la cooperación y el desarrollo, de modo que se pudieran establecer medidas concretas para su consecución y que pudieran ser evaluadas sobre la base de mecanismos comunes para cada uno de los países signatarios.

2. El establecimiento de un cronograma de cumplimiento de los distintos compromisos y su evaluación.

3. Aplicación del concepto de simetría, de tal manera que el conflicto centroamericano dejara de estar focalizado en un país de forma específica y se trabajara en la reconciliación nacional de cada país como parte de un proceso regional.

4. Aplicación del concepto de simultaneidad durante el desarrollo del proceso, lo que permitía la construcción de confianza entre las partes.

A medida que empezó a trascurrir el tiempo y cada uno de los países empezó a entrar en sus propias dinámicas, los conceptos del cronograma y el de simultaneidad fueron dejados de lado y el de simetría fue redefinido. Asimismo el de democracia fue tomando fuerza y el proceso se fue concentrando en Nicaragua. Es aquí, con el impulso del Procedimiento para establecer la Paz Firme y Duradera en Centroamérica, los instrumentos diseñados para su operativización y la Declaración de Costa del Sol, en El Salvador, que los acuerdos de tercera generación hacen su aparición y reclaman sus protagonismos.

Es importante recalcar que los Acuerdos de Esquipulas II y lo que se vendría posteriormente a lo interno de cada uno de los países Centroamericanos no hubiese sido posible sin el papel de la Perestroika
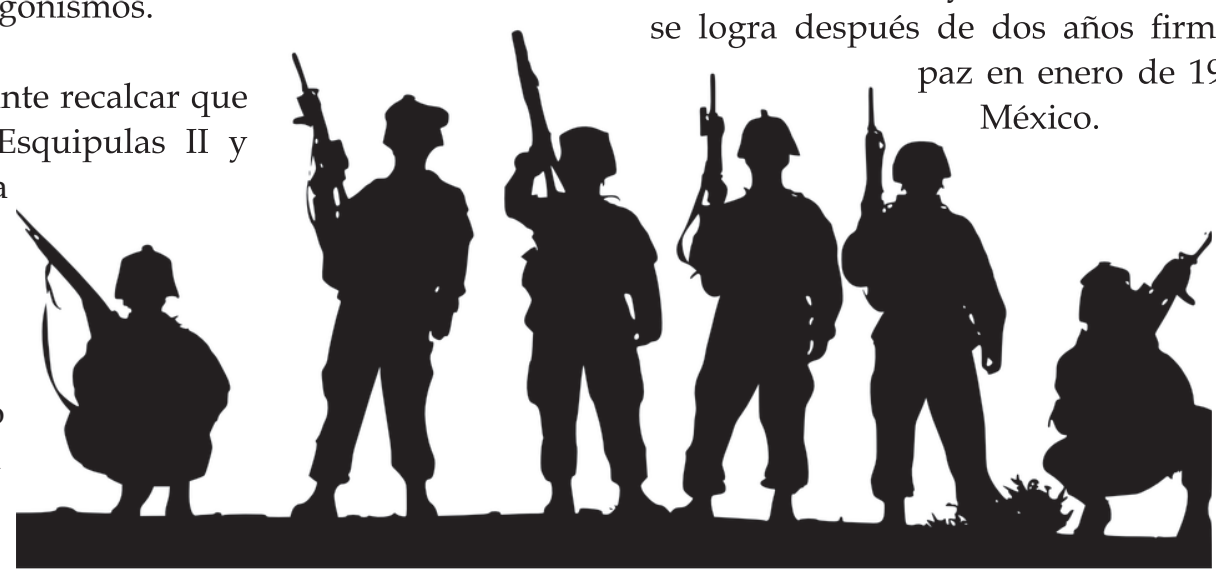

Pixabay.com por un lado y por el otro la falta de apoyo de los Demócratas en Estados Unidos a la continuidad de la Doctrina Reagan.

\section{Impacto de los Acuerdos de Esquipulas II en los Procesos de Pacificación de los Países Centroamericanos}

Como se ha venido comentando a los largo del documento, la implementación de los compromisos estipulados en los Acuerdos de Esquipulas II, se desarrolló de manera diferenciada en cada uno de los países que afrontaban conflictos internos, pues esto respondía a las propias dinámicas de negociación entre los bandos encontrados. En el caso de Nicaragua El cese al fuego se firmo en el año 1988, a través de la firma del Acuerdo de Sapoá y posteriormente en 1989 con los Acuerdos de Tela. Sin embargo no es hasta la firma del Acuerdo de Toncontin de 1990 cuando las condiciones para la total desmovilización de la Contra (Resistencia Nacional) tienen éxito.

En el caso de El Salvador, este proceso requirió de más tiempo y es así como en abril de 1990 mientras en Nicaragua se realiza un cambio de gobierno, electo por vía democrática y se desarrolla el proceso de desarme de la Resistencia Nicaragüense, el gobierno Salvadoreño y el FMLN reunidos en Suiza, apenas establecen los objetivos de la negociación de cara al proceso de paz. Después de una larga serie de negociaciones y acuerdos relativo a: la distribución de las tierras, modificación de las fuerzas armadas, la creación de una Policía Nacional Civil, la modificación al Sistema Judicial y a la defensa de los derechos humanos y modificaciones al Sistema Electoral, se logra después de dos años firmar los acuerdos de paz en enero de 1992 en Chapultepec 
la firma de los acuerdos de paz), y con una población más diversa, debido a su conformación étnica cultural. Dicha complejidad se vio manifestada en el periodo que necesito este país para llegar finalmente a la firma de los Acuerdos de Paz en Diciembre de 1996. Durante los 10 años posteriores a la firma de los Acuerdos de Esquipulas II, se desarrollaron diversas negociaciones entre el gobierno de Guatemala y la Unidad Revolucionaria Nacional Guatemalteca (URNG), dando como resultado la firma de 10 acuerdos que comprendían temas relativos a: Democratización, garantía del respeto de los derechos humanos, reconocimiento de los derechos de los pueblos indígenas, reforma agraria y la incorporación de la URNG a la vida política, entre otros, los que sumados daban contenido a la firma de los Acuerdos de Paz.

A manera de conclusión debe decirse que el Proceso de Paz en Centroamérica obedeció en primera instancia, a un esfuerzo latinoamericano que reconocía los problemas estructurales como las raíces de la violencia, pero también la capacidad de los centroamericanos de resolverlos sin intervención de terceros. En segundo lugar, al continuo esfuerzo de los países centroamericanos de crear medidas de confianza mutua, que les permitieran reconocerse como actores con demandas válidas y finalmente, el profundo interés de los bandos encontrados y de las sociedades centroamericanas de llegar a acuerdos negociados que permitieran mejorar sus condiciones de vida. Si bien en la actualidad se pueden encontrar diversas fallas, vacios, limitaciones e incluso retrocesos en el proceso de Centroamérica, no puede negarse la ausencia de la guerra y de sociedades en procesos de reconciliación, fin último de los Acuerdos de Paz, pero también el gran desafío de convertir esta paz en una cultura.

\section{Bibliografía}

Acta de Contadora Para la Paz y la Cooperación en Centroamérica (1986). Ciudad de Panamá.

http://www.acnur.org/t3/fileadmin/ Documentos/BDL/2002/1566.pdf?view $=1$

Alvarez, E. \& Palencia, T. (2002).Guatemala's peace process: context, analysis and evaluation.

Recuperado de: http://www.c-r.org/accord/ public-participation/guatemala-s-peace-processcontext-analysis-and-evaluation
Aravena Rojas, F. (1989, Marzo). El proceso de Esquipulas: El Desarrollo Conceptual y los Mecanismos Operativos. Universidad Nacional de Costa Rica. Trabajo Presentado en la Segunda Reunión Grupo de Trabajo Relaciones Internacionales de CLACSO Sobre la Cuenca del Caribe en Caracas, Venezuela. http://www.corteidh.or.cr/tablas/4693.pdf

Daniel, M. \& Ramírez, A. (S/F). El Proceso de Paz en Centroamérica. Agendas pendientes y nuevos focos de conflictividad: Los casos de Guatemala y Nicaragua. Global hoy. Recuperado de: http:// www.gloobal.net/iepala/gloobal/fichas/ficha.ph p?entidad $=$ Textos\&id $=9581 \&$ opcion $=$ documento

Díaz Barrado, M.C, Romero Serrano, J, Moran Blanco, S. (2010). Los conflictos armados de Centroamérica. Madrid. Ministerio de Defensa.

Duran, E. (S/F). La solución de Contadora para el logro de la paz en Centroamérica. Recuperado de: http://www.revistaei.uchile.cl/index.php/REI/ article/viewFile/15844/31710

Hidalgo Capitán, L. A. (2000). El cambio estructural del sistema socioeconómico costarricense desde una perspectiva compleja y evolutiva (1980-1998) (tesis inédita de doctorado). Universidad de Huelva. Huelva, España.

http://www.eumed.net/tesis-doctorales/ alhc/31.htm

Maira, L. (2014). Aprendizajes del Estudio de Estados Unidos. Programa de Estudios de Estados Unidos. Recuperado de:

https:/ / books.google.com.ni/books?id=PCPuBQ AAQBAJ\&pg=PT6\&dq=Aprendizajes+del+Estu dio+de+Estados+Unidos+fecha+del+libro\&hl= en\&sa $=$ X\&ved $=0$ CBsQ6AEwAGoVChMI35e $5 \mathrm{zb}$ HXyAIVCiseCh0TzAC5\# $\mathrm{v}=$ onepage\& $\mathrm{q}=$ Apren dizajes $\%$ 20del $\%$ 20Estudio $\% 20 \mathrm{de} \%$ 20Estados $\% 20$ Unidos\%20fecha\%20del\%20libro\&f=false

Van der Borgh, C. (S/F) El Salvador en transición. Rozemberg Quarterly. Recuperado de: http:// rozenbergquarterly.com/el-salvador-en-transicion-3/) 